\title{
Management of drug-resistant tuberculosis in special sub- populations including those with HIV co-infection, pregnancy, diabetes, organ-specific dysfunction, and in the critically ill
}

\author{
Aliasgar Esmail ${ }^{1 \#}$, Natasha F. Sabur ${ }^{1,2 \#}$, Ikechi Okpechi $^{3}$, Keertan Dheda $^{1,4}$ \\ ${ }^{1}$ Lung Infection and Immunity Unit, Division of Pulmonology and University of Cape Town Lung Institute, Department of Medicine, University of \\ Cape Town, Cape Town, South Africa; ${ }^{2}$ Division of Respirology, Department of Medicine, St. Michael's Hospital and West Park Healthcare Centre, \\ Toronto, Canada; ${ }^{3}$ Division of Nephrology, Department of Medicine, ${ }^{4}$ Institute of Infectious Diseases and Molecular Medicine, University of Cape \\ Town, Cape Town, South Africa \\ Contributions: (I) Conception and design: A Esmail, NF Sabur, K Dheda; (II) Administrative support: A Esmail, NF Sabur; (III) Provision of materials \\ or patients: All authors; (IV) Collection and assembly of data: None; (V) Data analysis and interpretation: All authors; (VI) Manuscript writing: All \\ authors; (VII) Final approval of manuscript: All authors. \\ \#These authors contributed equally to this work. \\ Correspondence to: Keertan Dheda. University of Cape Town, H47 Old Main Bldg, Groote Schuur Hospital, Observatory 7925, South Africa. \\ Email: keertan.dheda@uct.ac.za.
}

\begin{abstract}
Tuberculosis (TB) remains a major problem globally, and is the leading cause of death from an infectious agent. Drug-resistant TB threatens to marginalise the substantial gains that have recently been made in the fight against TB. Drug-resistant TB has significant associated morbidity and a high mortality, with only half of all multidrug-resistant TB patients achieving a successful treatment outcome. Patients with drug-resistant TB in resource-poor settings are now gaining access to newer and repurposed anti-TB drugs such as bedaquiline, delamanid and linezolid. However, with ever increasing rates of co-morbidity, there is little guidance on how to manage complex patients with drug-resistant TB. We address that knowledge gap, and outline principles underpinning the management of drug-resistant TB in special situations including HIV co-infection, pregnancy, renal disease, liver disease, diabetes, and in the critically ill.
\end{abstract}

Keywords: Drug-resistant tuberculosis (drug-resistant TB); HIV co-infection; bedaquiline; linezolid; delamanid

Submitted Jun 27, 2017. Accepted for publication Feb 25, 2018.

doi: $10.21037 /$ jtd.2018.05.11

View this article at: http://dx.doi.org/10.21037/jtd.2018.05.11

\section{Introduction}

Despite a slight decline in the global tuberculosis (TB) incidence over the last 13 years, there remains a substantial burden of disease with an estimated 10.4 million incident cases in 2015 (1). According to World Health Organization (WHO) estimates, 1.8 million people died from TB in the same year making TB the world's leading infectious cause of death (2).

TB diagnosis and management is complicated by drug resistance, which continues to threaten efforts at $\mathrm{TB}$ control because of its increasing burden in many countries (3), contributing to approximately $20 \%$ of global TB mortality (1), prohibitively high treatment costs $(4,5)$, and higher incidence rates in health care workers (6). Globally, there were an estimated 580,000 rifampicin resistant TB cases in 2015 (1). Approximately $10 \%$ of multi-drug resistant tuberculosis (MDR-TB) patients have extensively drug-resistant TB (XDR-TB), defined by resistance to rifampicin, isoniazid, fluoroquinolones and an injectable agent (7). While drug-sensitive new TB cases have a treatment success rate of $>85 \%$, drug-resistant $\mathrm{TB}$ patients fare poorly, with only $~ 50 \%$ of patients achieving 
Table 1 Medicines recommended for the treatment of rifampicin-resistant and multidrug-resistant $\mathrm{TB}^{1}$

\begin{tabular}{|c|c|c|c|c|}
\hline WHO class & \multicolumn{2}{|c|}{ Agent (abbreviation) } & Key toxicity & Comments \\
\hline $\begin{array}{l}\text { D. add-on agents } \\
\text { (not part of the core } \\
\text { MDR-TB regimen) }\end{array}$ & D2 & Bdq; delamanid (DIm) & $\begin{array}{l}\text { Bdq: QTc prolongation, } \\
\text { arthralgia, hepatitis and } \\
\text { headache; DIm: Hypokalemia, } \\
\text { nausea, vomiting, dizziness and } \\
\text { QTc prolongation }\end{array}$ & $\begin{array}{l}\text { Bdq/DIm: close monitoring of QTc is } \\
\text { recommended especially when using these } \\
\text { agents in combination with other QTc } \\
\text { prolonging drugs; Bdq: efavirenz (EFV) should } \\
\text { be changed to nevirapine (NVP) or a protease } \\
\text { inhibitor [may increase Bdq levels } \approx 2 \text {-fold } \\
\text { with unclear significance (11)], alternatively, } \\
\text { an integrase strand transfer inhibitor can be } \\
\text { used. Dlm: no significant anticipated drug- } \\
\text { drug interactions with ARVs (12) }\end{array}$ \\
\hline
\end{tabular}

${ }^{1}$ This regrouping is intended to guide the design of conventional regimens; ${ }^{2}$ Medicines in Groups A and C are shown by decreasing order of usual preference for use; 'Streptomycin may substitute other injectable agents under specific conditions. Resistance to streptomycin alone does not qualify for the definition of extensively drug-resistant TB (XDR-TB); ${ }^{4}$ Carbapenems and clavulanate are meant to be used together; clavulanate is only available in formulations combined with amoxicillin; ${ }^{5} \mathrm{HIV}$-status must be tested and confirmed to be negative before thioacetazone is started. ARVs, antiretroviral; TSH, thyroid stimulating hormone; CNS, central nervous system; QTc, corrected QT interval.

successful treatment (7). Pill burden, drug toxicity, and the duration of treatment contribute to high rates of loss to follow-up and a high mortality in this population (8).

Current WHO guidelines group treatment for rifampicin resistant anti-TB therapies into four classes (Table 1) (13) and provide principles for designing appropriate MDRTB treatment regimens (14). These regimens should include a minimum of four effective drugs (including an injectable agent) plus PZA which should be administered for an "intensive phase" of up to 8 months, followed by a "continuation phase" for minimum total treatment duration of 20 months $(14,15)$. More recently the WHO has recommended that, when certain pre-requisites can be fulfilled, a shorter MDR-TB regimen of 9-12 months can be used in selected patients (13), and repurposed drugs such as linezolid may be used where appropriate (13). 
The results of prospective outcome studies, including randomized controlled trials are underway (16). The WHO guidelines also recommend that when an effective drug regimen cannot be constituted because of drug toxicity or resistance, newer agents such as bedaquiline and delamanid may be used (13).

However, treatment of drug-resistant TB (DR-TB) remains challenging, and is more complex in patients with co-morbid disease where there is a limited evidence base to guide clinical practice. This review focuses on the challenges of treating drug-resistant TB in special populations, including in HIV-TB co-infected persons, pregnant women, patients with liver and renal dysfunction, diabetes, and those who are critically ill.

\section{Management of drug-resistant TB in patients co-infected with HIV}

Management of drug-resistant TB in the HIV-infected patient presents significant challenges due to shared toxicity between anti-HIV and TB drugs (Table 2). Greater potential for drug toxicity, HIV-related endorgan disease, pharmacokinetic drug-drug interactions, and immune reconstitution inflammatory syndrome (IRIS), especially involving the central nervous system, all contribute to the high mortality seen in MDR-TBHIV co-infected patients $(29,30)$.

New drugs used in the treatment of MDR-TB such as bedaquiline and linezolid have changed the face of MDR treatment; however, significant adverse event profiles require that these agents be used with caution, particularly in the setting of HIV where the potential for drug interactions is significant (see Table 2). The WHO has recently endorsed the use of dolutegravir as part of a first-line ART regimen (31). This agent is not only more effective and better tolerated, but is also expected to be safe for co-administration with newer agents such as bedaquiline and delamanid $(32,33)$.

\section{Timing of initiating antiretroviral therapy (ART) in drug-resistant TB-HIV co-infection}

Antiretroviral therapy improves survival in patients with MDR-TB $(29,34,35)$. The WHO recommends that newly diagnosed MDR-TB/HIV co-infected patients should initiate ART within the first 8 weeks of starting effective MDR-TB treatment irrespective of CD4+ count (36). As with drug-sensitive TB, patients with CD4+ counts
$<50$ cells $/ \mathrm{mm}^{3}$ should initiate ARVs within 2 weeks of starting MDR-TB treatment (37-39), unless if they are suspected to have TB meningitis (in which case initiation of ARVs should be deferred due to the risk of developing potentially fatal IRIS) $(40,41)$.

\section{Bedaquiline (Bdq) use in the drug-resistant TB-HIV co-infected patient}

Bedaquiline (trade name Sirturo) is a diarylquinoline and the first new anti-TB drug on the market in over 40 years. This orally administrated medication acts via a novel mechanism that selectively inhibits mycobacterial adenosine triphosphate synthase $(42,43)$. Bedaquiline is known to cause prolongation of the QTc interval, and while a phase IIB trial demonstrated higher rates of culture conversion in the bedaquiline arm, there were also more deaths in those receiving the study drug (44). Although this was not felt to be drug related, bedaquiline use warrants regular ECG monitoring.

Bedaquiline is well tolerated (45) and has demonstrated both safety and efficacy in HIV co-infected patients (46). The WHO-recommended first-line antiretroviral therapy (ART) regimens include nevirapine and efavirenz, both of which induce CYP3A4, and the second-line regimens include ritonavir-boosted protease inhibitors, which inhibit CYP3A4. Drug-drug interaction studies of HIVinfected patients on steady state ART, where a single dose of Bdq was given, found that nevirapine, lopinavirritonavir (LPV/r), and efavirenz were associated with no effect, an increase (significance unclear), and a decrease in the area under the curve, respectively (47). However, single doses of bedaquiline may under-estimate the magnitude of interactions experienced when bedaquiline reaches steadystate. Population pharmacokinetic studies modelling the data from single dose Bdq and antiretroviral drug-drug interaction studies estimated the following changes in Bdq exposure at steady-state: $\mathrm{LPV} / \mathrm{r}$ - 3 -fold increase; efavirenz-decreased by $52 \%$; nevirapine-no significant effect $(11,48)$. A study of patients on ART being treated with Bdq for drug-resistant TB confirmed the findings of the population pharmacokinetic studies on interactions with nevirapine and LPV/r (49). Bdq should not be used with efavirenz, and caution needs to be exercised when using with $\mathrm{LPV} / \mathrm{r}$, with close monitoring for Bdq toxicity. Other potential ART options for co-administration with Bdq are the integrase strand transfer inhibitors raltegravir or dolutegravir together with dual nucleoside reverse 
Table 2 Shared toxicity between anti-TB therapy and antiretroviral agents

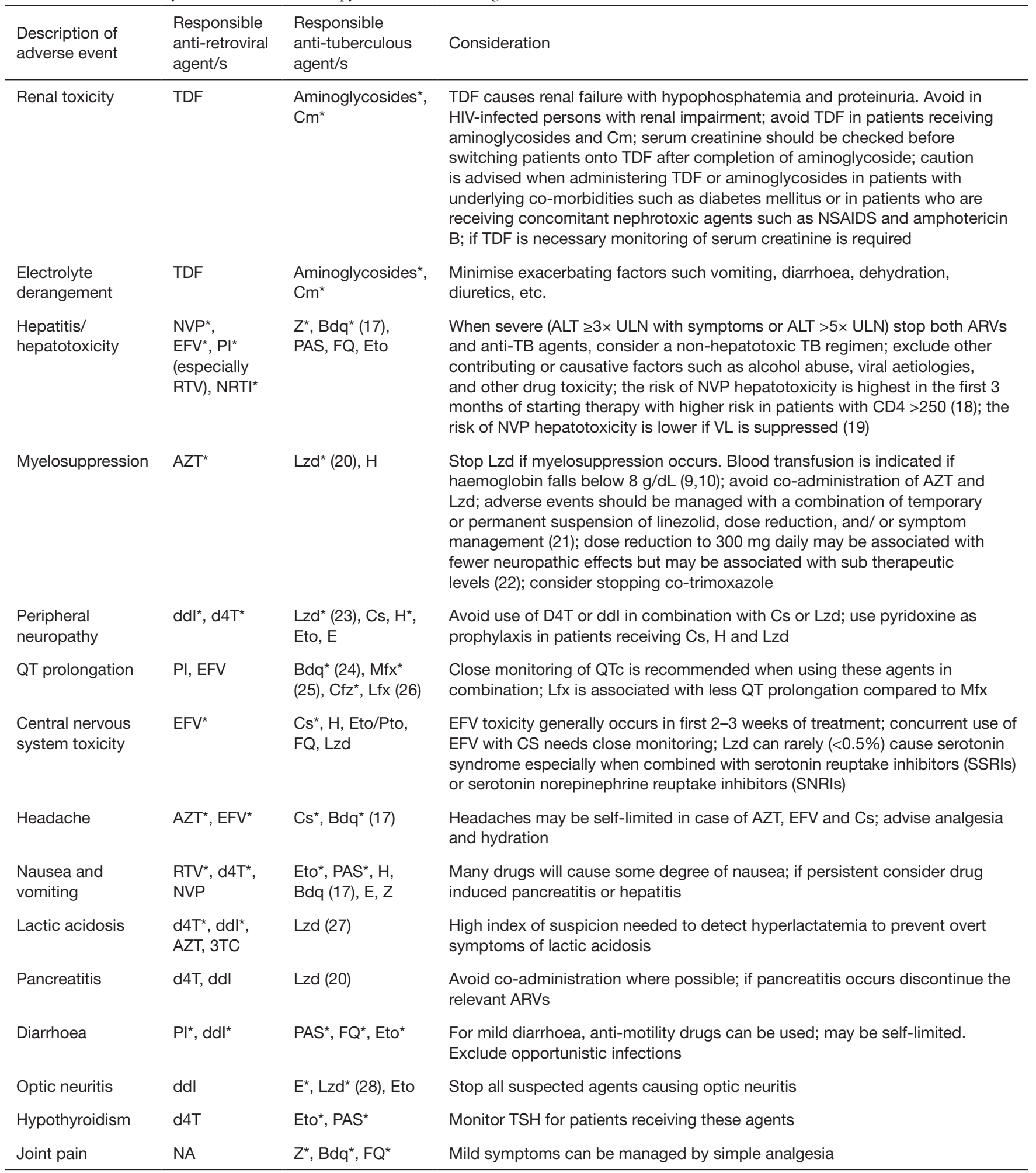

${ }^{*}$ Drugs most likely to contribute towards toxicity. $\mathrm{Cm}$, capreomycin; $\mathrm{H}$, isoniazid, PAS, para-amino salicylic acid; Z, pyrazinamide; Bdq, bedaquiline; Lzd, linezolid; Eto, ethionamide; Pto, prothionamide; FQ, fluoroquinolones; Mfx, moxifloxacin, Lfx, levofloxacin E, ethambutol; Cs, Cycloserine; TDF, tenofovir; EFV, efavirenz; ddl, didanosine; d4T, stavudine; PI, protease inhibitor; 3TC, lamivudine; NVP, nevirapine; RTV, ritonavir; AZT, zidovudine; QTc, corrected QT interval; TSH, thyroid stimulating hormone; CNS, central nervous system. 
transcriptase inhibitors (NRTIs); however, these agents are not widely available in many resource-limited settings. In settings where integrase strand transfer inhibitors are unavailable, and where an effective ART regimen cannot otherwise be constructed, the use of triple NRTIs may be an option for co-administration with Bdq. However, this regimen should only be used after virologic suppression has been achieved (50).

\section{Use of linezolid and other oxazolidinones in the drug- resistant TB-HIV co-infected patient}

Linezolid, an oxazolidinone, binds to the $70 \mathrm{~S}$ initiation complex of bacterial ribosomes and disrupts protein synthesis. It is orally administered and has demonstrated effective anti-mycobacterial activity in vitro and in animal studies. Case series and several systematic reviews have shown that MDR and XDR-TB regimens incorporating linezolid have improved clinical outcomes $(23,51,52)$, and high culture conversion rates have been reported in compassionate use programs in South Africa where $56 \%$ of the cohort was HIV co-infected (46).

There is potential for significant worsening of HIVrelated neuropathy and bone marrow dysfunction in the presence of linezolid, particularly anaemia when coadministered with zidovudine, and peripheral neuropathy when combined with stavudine (29). Provided these red flags are considered, linezolid can be used for MDR-TB treatment in the HIV co-infected patient with careful monitoring. While $600 \mathrm{mg}$ daily is the standard dose of linezolid in drug-resistant TB regimens, $300 \mathrm{mg}$ daily may reduce potential for adverse events (22). However, acquired drug resistance is more likely with a lower starting dose (52). Further studies are needed to determine the optimal dose to maintain culture conversion, minimize adverse events, and prevent amplification of resistance.

Several retrospective case series of HIV-infected patients with MDR and XDR-TB receiving linezolid as part of their regimen have demonstrated promising culture conversion rates, but high rates of associated drug-related adverse events, mainly due to peripheral neuropathy, anemia, and optic neuritis (53). However, improved treatment outcomes with linezolid may justify the use of this drug in patients co-infected with HIV. Therapeutic drug monitoring using dried blood spot analysis (54), measuring plasma concentrations (55) and measuring the cytochrome c oxidase/citrate synthase activity ratio from peripheral blood (56), if available, could play a helpful role in maximizing efficacy of linezolid, while limiting toxicity, but prospective studies are required. HIV-infected patients receiving linezolid should receive pyridoxine prophylaxis, particularly when linezolid is co-administered with other potentially neurotoxic drugs such as high-dose isoniazid (especially in MDR-TB patients with inhA mutations where high dose isoniazid is being used). Peripheral neuropathy often occurs after 3 months of treatment (57). These patients must be monitored closely for subjective neuropathy symptoms as well as by neurological assessment for peripheral neuropathy including measurement of vibration sense at each follow up visit. In the event of bone marrow suppression, a dose reduction can be attempted in addition to correcting other contributory factors such as concomitant nutritional deficiency and cessation of other myelotoxic drugs (such as sulfamethoxazole and trimethoprim); if blood counts do not improve, linezolid should be discontinued.

Patients with renal dysfunction who are receiving linezolid with potentially myelosuppressive ARVs are especially at risk for thrombocytopenia and dose adjustment should be considered in patients with creatinine clearance of $<30 \mathrm{~mL} / \mathrm{min}(58,59)$. The major mechanism of linezolidassociate neuropathy is mitochondrial toxicity, since linezolid interferes with mitochondrial protein synthesis $(60,61)$. If peripheral neuropathy develops while on linezolid, dose reduction may be considered depending in the clinical context including rapidity of progression, severity, regimens. Thus, if neuropathy is minimal and stabilizes upon dose reduction, linezolid may be continued in select cases (62). Any evidence of optic neuritis warrants immediate discontinuation of linezolid, as this is usually irreversible (63). Close follow up with serial monthly visual acuity measurements and colour vision assessment is advised when using linezolid together with ethambutol (64).

Linezolid, whilst effective in the treatment of DR-TB, has substantial myelotoxicity and neurotoxicity resulting in almost a third of requiring dose reduction or discontinuation of linezolid altogether (65). By contrast, tedizolid, a newer oxazolidinone currently registered for skin and soft tissue infections in the USA and European Union (66), is less myelotoxic than linezolid in short-term studies (67). Animal studies have demonstrated no evidence of neuropathy $(68,69)$. Population based pharmacokinetic models have shown that over the course of a dosing interval (using standard therapeutic doses), free plasma concentrations of tedizolid fell below the toxic concentrations for causing mitochondrial protein synthesis suppression in $84 \%$ (70) 
vs. $38 \%$ of linezolid-treated patients $(56,71)$. Tedizolid, in the hollow fibre model, has shown anti-tuberculous efficacy equivalent to that of rifampicin and moxifloxacin. Clinical trials in patients with DR-Tb are now warranted.

Sutezolid is another oxazolidinone that is efficacious against $M . t b$ in rat models where it shortened treatment by 1 month compared to linezolid, which failed to show a treatment shortening effect (72). The maximum bactericidal activity of sutezolid is estimated to be twice that of linezolid, with a favourable safety profile in whole blood culture models (73). Furthermore, sutezolid has bactericidal activity against both intra and extracellular mycobacteria (74). In a phase 1 study sutezolid has been shown to be potentially safer than linezolid when given to healthy volunteers at a dose of $600 \mathrm{mg}$ twice daily for 28 days with no reports of hematologic, neurologic or biochemical toxicity (73). However, more recently, 6-month rat toxicity studies have raised concerns about the longer term safety profile of sutezolid. Both tedizolid and sutezolid are compatible with antiretrovirals and other TB drugs. Collectively, the available evidence suggests that these agents may be more efficacious and have a lower adverse event rate, but this requires confirmation in phase 2 and 3 studies pending safety clarifications about sutezolid.

\section{Delamanid use in the drug-resistant TB-HIV co-infected patient}

Delamanid is an orally administered agent that acts by inhibiting mycolic acid synthesis in the mycobacterial cell wall (75). It has demonstrated promising results in drugresistant $\mathrm{TB}$, showing increased rates of sputum culture conversion and improved clinical outcomes (76-78). Phase III clinical trials have been completed but, disappointingly, the delaminid failed to show improved outcomes when added to an optimized background regimen. Clinical drug-drug interaction studies have been performed on healthy volunteers and co-administration with tenofovir and efavirenz did not affect delamanid exposure, but lopinavir/ritonavir co-administration significantly increased delamanid exposure (79). These results are of uncertain significance.

Delamanid is known to cause prolongation of the QTc interval via the DM-6705 metabolite, and therefore regular ECG monitoring is required. Formation of the DM-6705 metabolite is regulated by serum albumin, and delamanid is consequently contraindicated in hypoalbuminemia (albumin $<28 \mathrm{~g} / \mathrm{L}$ ), which is a frequent finding in HIV-infected individuals, particularly in advanced disease, and may be a predictor for progression to AIDS and death $(80,81)$. Low serum albumin may also be more commonly found in patients with TB-HIV co-infection (82). Unfortunately, this may limit delamanid's use in the HIV co-infected population despite having a safe drug-drug interaction profile with first-line antiretroviral medications (83). Additionally, delamanid is administered as twice daily dosing, 30-minutes after a standard meal (84). This twicedaily dosing may impact adherence (85) and require additional resources to ensure drug administration under observation. Delamanid is potentially efficacious and well tolerated in children aged 6 years and older (86), however controlled studies are lacking in the pediatric population.

\section{Management of drug-resistant TB in pregnancy}

The burden of TB in pregnant women is substantial (87), with prevalence estimates amongst pregnant and post-partum women ranging from $0.06 \%$ to $7.2 \%$, and as high as $11 \%$ in HIV-infected women in a highburden setting (87-89). Women may be more vulnerable to $\mathrm{TB}$ disease during pregnancy and in the post-partum period. T-helper 1 (Th-1)/Th-2 ratio is reduced during pregnancy, thus potentially increasing susceptibility to new infection and reactivation of TB. In the early post-partum period, Th-1 suppression may be reversed (88). This may be associated with exacerbation of symptoms akin to the immune reconstitution syndrome seen in HIV patients after ART therapy is commenced (90). TB during pregnancy is also associated with poor outcomes, including an increased risk of preterm birth, low birth weight, intrauterine growth restriction, and perinatal death $(91,92)$.

A decision on the timing of treatment for drug-resistant $\mathrm{TB}$ and the construction of a drug-resistant TB regimen during pregnancy should take into consideration the gestational age of the foetus, and should weigh the risks of the teratogenic effects of anti-TB treatment carefully against potential benefit to the mother (14). Ideally, all pregnant women should be started on treatment as soon as possible. However, as most teratogenicity occurs during the first trimester, in selected cases where the clinical condition of the mother is stable and where there is minimal radiological disease, treatment may be deferred until the second trimester. This strategy must be accompanied by close clinical follow-up as drug-resistant TB in pregnancy can have an accelerated course.

Guidance for treatment of MDR-TB in pregnancy 
Table 3 FDA based classification of drugs used for MDR-TB treatment during pregnancy

\begin{tabular}{lc}
\hline MDR-TB drug & FDA classification \\
\hline Pyrazinamide & $\mathrm{C}$ \\
Fluoroquinolones: levofloxacin; & $\mathrm{C}$ \\
moxifloxacin; ofloxacin & \\
Aminoglycosides: kanamycin; & $\mathrm{D}$ \\
amikacin; streptomycin & \\
Capreomycin & $\mathrm{C}$ \\
Ethionamide/prothionamide & $\mathrm{C}$ \\
Cycloserine/terizidone & $\mathrm{C}$ \\
Para-aminosalicylic acid & $\mathrm{C}$ \\
High-dose isoniazid & $\mathrm{C}$ \\
Clofazimine & $\mathrm{C}$ \\
Linezolid & $\mathrm{C}$ \\
Amoxicillin/clavulanate & $\mathrm{B}$ \\
Clarithromycin & $\mathrm{C}$ \\
Imipenem/cilastatin & $\mathrm{C}$ \\
Meropenem & $\mathrm{B}$ \\
Bedaquiline & $\mathrm{B}$ \\
Delamanid & Not FA approved \\
\hline
\end{tabular}

FDA pregnancy categories: A-human studies demonstrate no risk; B-animal studies demonstrate no risk, no human studies; C-animal studies demonstrate risk, no human studies; $\mathrm{D}$-human studies demonstrate risk; $\mathrm{X}$-contraindicated in pregnancy.

comes only from case reports and case series. While some women choose to terminate pregnancy due to the possible teratogenicity of anti-TB treatment, case series suggest that favourable outcomes are achievable $(93,94)$. In the largest series of MDR-TB treatment in pregnancy $(n=38), 60 \%$ of patients were cured, $21 \%$ of patients had pregnancyassociated complications including vaginal bleeding and spontaneous abortion, and no teratogenic effects were seen in the infants (94).

Ideally, the regimen for MDR-TB in pregnancy will consist of at least four second-line anti-TB drugs that are likely to be effective against the infecting strain, plus pyrazinamide. Most of the second line anti-TB agents are pregnancy class $C$ per the US Food and Drug Administration (FDA; Table 3). Aminoglycosides, specifically amikacin and kanamycin, are FDA class D, and should be excluded from TB treatment regimens during pregnancy, especially within the first 20 weeks, because of the risk of ototoxicity and foetal malformation. In selected cases with severe disease with no available alternatives, these agents can be used with close monitoring but should be deferred until after 20 weeks (95). Capreomycin, a polypeptide with similar activity to aminoglycosides, is an FDA class $\mathrm{C}$ drug with a reduced toxicity profile. In severe cases of multidrug-resistant TB where aminoglycoside treatment must be given at the outset, capreomycin is the injectable agent of choice (95), and can be given thrice weekly to decrease drug exposure to the foetus. Ethionamide is generally avoided as it can increase the risk of nausea and vomiting associated with pregnancy. These drugs can be reintroduced after delivery to strengthen the regimen in the immediate postpartum period. The WHO has not recommend the use of bedaquiline and delamanid in pregnancy due to the lack safety and efficacy data (96). Delamanid, in animal studies, has been shown to be potentially teratogenic and should be avoided until more data is available (97) whereas bedaquiline has been demonstrated to be safe in animal reproduction studies (category B), and may be considered for individual women who have contra-indications to aminoglycoside use or in whom an effective regimen cannot otherwise be constructed $(24,98)$. With regards to breast feeding, smear positive mothers should discontinue breast feeding if possible. Both bedaquiline and delamanid are excreted in breast milk in animal studies and therefore, the decision to discontinue the drug or nursing, as an alternative, should depend on the clinical context.

Lastly, in view of the toxic effects of MDR-TB drugs to the expectant mother and the foetus, it is critically important to offer individualised, long term and effective contraception (e.g., Depo-Provera or an intra-uterine contraceptive device) to all women of child bearing age who are receiving treatment for DR-TB.

\section{Management of drug-resistant TB in patients with renal impairment}

Renal failure may be due to a concomitant medical problem or may be a result of previous treatment for DR-TB with an aminoglycoside. Dosing of drugs should be adjusted per patient's creatinine clearance (an estimate of the glomerular filtration rate) (Table 4). For several drugs, the WHO has suggested that the dose and/or the interval between dosing should be adjusted for patients with creatinine clearance 
less than $<30 \mathrm{~mL} / \mathrm{min}$ or those receiving haemodialysis (14). While some clinicians have had to previously persist with thrice-weekly dosage of aminoglycosides because of the lack of alternative options, the availability of newer drugs such as bedaquiline should render this practice obsolete. In clinical practice, it is now not uncommon to use Bdq when there is aminoglycoside-associated toxicity.

Tenofovir, an antiretroviral agent, can cause renal dysfunction and severe electrolyte wasting with lifethreatening hypokalaemia when administered with aminoglycosides or capreomycin as both concentrate in the proximal convoluted tubules; co-administration should be avoided (110-112) (see Table 1). In rare cases where this combination cannot be avoided, special emphasis must be placed on correction of underlying factors that may potentiate toxicity such as diarrhoea, dehydration, diuretic use and concomitant administration of other nephrotoxic medications (113). Co-administration of these agents also warrants close monitoring of creatinine, serum potassium and aminoglycoside drug levels if available; in the event of electrolyte wasting or worsening renal dysfunction, both drugs should be stopped until the patient is electrolyte replete. In patients of African heritage, the risks of iatrogenic nephrotoxicity may be greater due to the background prevalence of HIVAN (HIV associated nephropathy) $(113,114)$. In such patients, the use of tenofovir and/or aminoglycoside should be avoided, especially, in patients with advanced HIV $(115,116)$ and nephrotic range proteinuria $(115,117)$.

\section{Management of drug-resistant TB in patients with liver dysfunction}

Although technically a first-line drug, pyrazinamide is the most hepatotoxic agent employed in the DR-TB regimen. Patients with significant chronic liver disease should not receive pyrazinamide. Isoniazid has been used high doses (16-18 mg/kg) in cases of MDR-TB with low-level INH resistance (conferred by the $i n h A$ mutation), and although no evidence of a greater likelihood of acute hepatotoxicity was found in patients with normal liver function (118), its use in patients with concomitant stable chronic liver disease at high doses has not been studied. We recommend that it should be excluded from MDR-TB regimens in patients with chronic liver disease except in selected cases. Of the second-line drugs, ethionamide, prothionamide and PAS can also be hepatotoxic, while the fluoroquinolones are rarely implicated in hepatitis.
All second-line drugs can be used in chronic stable liver disease, but close monitoring of liver enzymes is mandatory, and significant deterioration in liver function should trigger immediate withdrawal of the offending drug. The source of other causes for liver dysfunction, including viral hepatitis and alcohol consumption, should be addressed and treated to prevent further complications during treatment. In cases of acute hepatitis, anti-TB treatment should be deferred until hepatitis has stabilized. When formulating a regimen in patients with chronic liver dysfunction, a combination of 4 non-hepatotoxic drugs should ideally be used with the inclusion of a fluoroquinolones to ensure efficacy of the regimen (14).

In patients receiving $\mathrm{TB}$ treatment, chronic hepatitis $\mathrm{B}$ infection is considered a risk factor for hepatotoxicity (119). Patients who are sero-positive for hepatitis B e antigen are more prone to hepatotoxicity compared to patients who are hepatitis e antigen negative (120). If treatment for hepatitis B infection is indicated, ART should be initiated with the combination of at least two agents active against hepatitis B, e.g., tenofovir and emtricitabine or lamivudine $(121,122)$. Entecavir can be considered in place of tenofovir in patients with renal dysfunction (dose adjustment may be required) (122). In HIV/HCV co-infection drug interactions and overlapping toxicities with MDR-TB agents must be considered (121).

\section{Management of drug-resistant TB in patients with diabetes mellitus}

The prevalence of diabetes mellitus has increased substantially worldwide, with an estimated 415 million adults suffering from diabetes in 2015. The rates of type II diabetes also appear to be increasing in developing regions such as sub-Saharan Africa and India (123). Diabetes mellitus confers a 3 -fold increased risk of developing active TB $(124,125)$, and is associated with worse TB treatment outcomes, including delayed sputum culture conversion, and higher rates of treatment failure, relapse and recurrence (126-128). Furthermore, TB itself can induce glucose intolerance resulting in worsening glycaemic control (129).

Preliminary evidence suggests that that there may be an association between diabetes and MDR-TB (130,131). More recently, diabetes has also been shown to increase the risk of primary infection with MDR-TB and is associated with delayed sputum conversion $(132,133)$. Evidence is emerging to suggest that diabetes mellitus may also play a role in the development of drug resistant TB $(134,135)$. 
Table 4 Dosing of MDR-TB agents in patients with renal impairment

\begin{tabular}{|c|c|c|c|}
\hline $\begin{array}{l}\text { Mycobacterial agent } \\
\text { and usual dose }\end{array}$ & $\begin{array}{l}\text { Degree of renal } \\
\text { impairment (Cr clearance) }\end{array}$ & $\begin{array}{l}\text { Renal replacement } \\
\text { therapy }\end{array}$ & Special prescriber points \\
\hline $\begin{array}{l}\text { Aminoglycosides } \\
(99-101)(\mathrm{Cm}, \mathrm{Km} \text {, } \\
\text { Am) } 15-20 \mathrm{mg} / \mathrm{kg}\end{array}$ & $\begin{array}{l}\text { Dosing should be } \\
\text { adjusted to achieve } \\
\text { undetectable plasma } \\
\text { trough levels at all levels } \\
\text { of renal impairment }\end{array}$ & $\begin{array}{l}\text { Dosing should be } \\
\text { adjusted to achieve } \\
\text { undetectable plasma } \\
\text { trough levels }\end{array}$ & $\begin{array}{l}\text { Must be AVOIDED if possible; main route of clearance } \\
\text { is renal; } 3 x / \text { week vs. daily has no difference in oto/ } \\
\text { nephrotoxicity; dose adjustment and therapeutic drug } \\
\text { monitoring required due to toxicity risk and changes } \\
\text { in drug clearance over time; monitoring should include } \\
\text { regular U\&Es to assess renal function along with clinical } \\
\text { assessment, audiometry }\end{array}$ \\
\hline
\end{tabular}

Pyrazinamide
$(102-104)$
$25-30 \mathrm{mg} / \mathrm{kg} / \mathrm{day}$
$1.5 \mathrm{~g}$ for $50 \mathrm{~kg}, 2 \mathrm{~g}$
for $>50 \mathrm{~kg}$
Ethambutol
(103-105),
$15-25 \mathrm{mg} / \mathrm{kg} /$ day (103)

Ethionamide (106), $15-20 \mathrm{mg} / \mathrm{kg} /$ day in divided doses

Cycloserine (106), $10-15 \mathrm{mg} / \mathrm{kg} /$ day in divided doses

Para-aminosalicylic acid (PAS) (106) 8-12 g/day

Linezolid $600 \mathrm{mg} / \mathrm{day}$

Isoniazid $(103,107)$, $16-18 \mathrm{mg} / \mathrm{kg}$

Clofazamine (106), $100 \mathrm{mg}$ daily

Fluoroquinolones (108) moxifloxacin $400 \mathrm{mg}$ daily; levofloxacin $(108,109)$ 750-1,000 mg daily $\geq 30 \mathrm{~mL} / \mathrm{min}$ : no dose adjustment required; $<30 \mathrm{~mL} / \mathrm{min}$ : 25-30 $\mathrm{mg} / \mathrm{kg}$ three times per week

$\geq 30 \mathrm{~mL} / \mathrm{min}$ : No dose adjustment required; $<30 \mathrm{~mL} / \mathrm{min}: 15-25 \mathrm{mg} / \mathrm{kg}$ three times per week

No dose adjustment required

$\geq 30 \mathrm{~mL} / \mathrm{min}$ : no dose adjustment required; $<30 \mathrm{~mL} / \mathrm{min}$ : $250 \mathrm{mg}$ daily or $500 \mathrm{mg}$ alternate days

No dose adjustment required

No dose adjustment required

No dose adjustment required

No dose adjustment needed

Moxifloxacin: no dose adjustments needed; levofloxacin: 30-50 $\mathrm{mL} / \mathrm{min}$ (750-1,000 mg), $<30 \mathrm{~mL} / \mathrm{min}$ (750-1,000 mg three times per week)
25-30 mg/kg three times/week after dialysis

15-25 mg/kg three times a week; dialysis does not eliminate drug significantly

No dose adjustment required

$250 \mathrm{mg}$ daily or $500 \mathrm{mg}$ alternate days given after hemodialysis

Hemodialysis eliminates it's the active metabolite. Dosing should therefore be given post dialysis

No dose adjustments required

No dose adjustments required; hemodialysis does not eliminate drug significantly

No dose adjustment needed

Moxifloxacin: no dose adjustments necessary; levofloxacin: 750-1,000 mg alternate days
Can be used safely in renal disease; main route of clearance is hepatic with active metabolites undergoing some renal clearance; monitor LFTs for hepatotoxicity; monitor for gout due to reduced uric acid clearance in renal failure

AVOID use if possible; main route of clearance is renal; ocular toxicity is a significant concern in patients with renal disease

Main route of clearance is hepatic; monitor for neuropathy and hepatotoxicity

AVOID if possible in renal disease. As the main route of clearance is renal; Increased risk of significant neurotoxicity; plasma level monitoring should be used if available

Use with extreme caution in renal disease as the main route of clearance is renal; increased risk of acidosis and gastrointestinal side effects

Main route of clearance is hepatic with some renal clearance; increased risk of haematological toxicity and peripheral neuropathy

Co-administer with pyridoxine

Monitor QTc when used concurrently with other QTC prolonging agents like bedaquiline, delamanid and fluoroquinolones in patients with renal insufficiency

Moxifloxacin is predominantly cleared by the hepatobiliary route while levofloxacin has significant renal clearance in addition; there may be a higher risk of neurotoxicity and tendinopathies when using fluoroquinolones in advanced renal insufficiency; avoid concomitant administration of antacids, phosphate binders, calcium, iron or aluminium containing medications to avoid mal-absorption 
The thresholds for the diagnosis of diabetes in patients with DR-TB/HIV co-infection remain the same as the general population (136), however, special considerations need to be afforded when using $\mathrm{HbA1c}$, which may underestimate poor glycaemic control (HbA1C may be modulated by HIV that decreases red blood cell life span, NRTI use may increase mean corpuscular volume, and results are discordant at CD 4 counts $(<500$ cells/mL) (137-139). Thus, fasting plasma glucose (FPG) may be more in this population.

Rifampicin, when used for drug sensitive TB, enhances the metabolism of sulphonylureas (140), and when co-administered with metformin may exaggerate hypoglycaemia via increased expression of organic cation transporter (OCT-1) (141). Newer anti-diabetic agents such as glucagon-like peptide-1 receptor agonists and dipeptidyl peptidase-4 inhibitor are potentially safer when co-administered with rifampicin for drug-sensitive or INH-monoresistant TB (141). The optimal dose for rifamycins is currently under review and several studies are evaluating the impact of increased rifamycin dosage (142), which may further amplify these effects. Additionally, metformin may induce more pronounced gastrointestinal side-effects when co-administered with anti-tuberculous agents such as ethionamide, para-amino salicylic acid and clofazimine, and can rarely cause lactic acidosis (143). Caution should be instituted when using nephrotoxic agents such as aminoglycosides, and neurotoxic agents such as linezolid, in patients with established diabetes. QTc monitoring is advised when using bedaquiline and/ or delamanid concurrently with hypoglycaemic agents such sulphonylureas and glinides since these agents' function by inhibiting ATP-dependent potassium channels thus delaying repolarization which leads to prolongation of QTc (144). Furthermore, special consideration also needs to be afforded when using bedaquiline with potentially hepatotoxic hypoglycaemic agents such as thiazolidinediones (145).

The pharmacological management drug-resistant TB remains similar for diabetic and non-diabetic patients; however, this may need reconsideration in view of the increased treatment failure rates seen in patients with uncontrolled diabetes $(146,147)$. Optimal glycaemic control may result in better outcomes. It is therefore recommended that diabetic patients are treated aggressively to achieve optimal control (148). The use of modern insulin or insulin analogues must be utilized where necessary, especially in the early phase of TB treatment (149), however, caution needs to be exercised when treating patients with tight glycaemic control, and to ensure that health systems are well equipped to monitor the patient and prevent adverse events such as hypoglycaemia (150). Furthermore, patients with TB may have poor appetite due to TB itself or due to the adverse effects of the various anti-TB agents. This may cause marked changes in weight (initially weight loss followed by weight gain during TB treatment), which could potentially cause further problems with optimal dosing of antiglycaemic agents. Patients with diabetes are known to have increased rates of TB relapse and recurrence $(127,151,152)$, and should therefore be appropriately counselled and followed up.

\section{Management of drug-resistant TB in the intensive care unit}

In high-burden settings, TB is frequently diagnosed in the ICU, even in patients without a respiratory indication for admission such as trauma or emergency surgery (153). A proportion of these patients will have drug-resistant TB. The management of drug-resistant TB in the ICU is challenging, complicated by pharmacokinetic concerns such as poor gastric absorption, high rates of organ dysfunction, and drug toxicity. Concomitant renal failure precludes the use of aminoglycosides. Therapeutic drug monitoring (TDM) should be used whenever possible, and may timely adjustment of drug therapy $(154,155)$. Rifampicin absorption is often stochastic and preliminary studies demonstrate that levels are often very low in patients admitted to the intensive care unit (156). TDM for second-line agents such as linezolid, fluoroquinolones and injectable drugs is expensive and not widely available (156). Newer methods such as utilization of dried blood spot (DBS) may overcome logistical challenges, thereby making TDM for drugs like linezolid and moxifloxacin more cost effective.

Infection control is critical and patients should be isolated in individual negative-pressure rooms, and managed with an endotracheal suctioning system without disconnection (closed suctioning) and a bacterial (HME) filter in the expiratory limb of the ventilator circuit. Appropriate infection control precautions should be observed, and during or after high-risk situations such as endotracheal tube changes or during extubation.

\section{Conclusions}

Access to more accurate diagnostic techniques, including Xpert MTB/RIF Ultra testing, is continually improving, even in resource-poor areas. This promises to enhance 
detection rates of multi-drug resistant TB. Better detection of drug-resistance combined with ever increasing rates of co-morbid disease means that increasing numbers of more complex cases are likely to be seen. This review provides guidance on management of drug-resistant TB in special situations including co-morbid disease, pregnancy, and critical illness. However, good evidence to support management decisions in these sub-groups is scanty as these patients are often excluded from clinical trials. Further research is necessary to guide practice and support clinical decision-making in these patient sub-groups, and in different clinical settings (including those that are resource constrained).

\section{Acknowledgements}

South African Medical Research Council (SA MRC); European and Developing Countries Clinical Trials Partnership (EDCTP).

\section{Footnote}

Conflicts of Interest: The authors have no conflicts of interest to declare.

\section{References}

1. Global tuberculosis report 2016. Geneva: World Health Organization; 2016.

2. Dheda K, Barry CE 3rd, Maartens G. Tuberculosis. Lancet 2016;387:1211-26.

3. Gandhi NR, Nunn P, Dheda K, et al. Multidrug-resistant and extensively drug-resistant tuberculosis: a threat to global control of tuberculosis. Lancet 2010;375:1830-43.

4. Marks SM, Flood J, Seaworth B, et al. Treatment practices, outcomes, and costs of multidrug-resistant and extensively drug-resistant tuberculosis, United States, 2005-2007. Emerg Infect Dis 2014;20:812-21.

5. Pooran A, Pieterson E, Davids M, et al. What is the cost of diagnosis and management of drug resistant tuberculosis in South Africa? PLoS One 2013;8:e54587.

6. O'Donnell MR, Jarand J, Loveday M, et al. High incidence of hospital admissions with multidrug-resistant and extensively drug-resistant tuberculosis among South African health care workers. Ann Intern Med 2010;153:516-22.

7. WHO. Global Tuberculosis Report. Geneva, Switzerland: World Health Organization, 2015.
8. Dheda K, Gumbo T, Maartens G, et al. The epidemiology, pathogenesis, transmission, diagnosis, and management of multidrug-resistant, extensively drug-resistant, and incurable tuberculosis. Lancet Respir Med 2017.

9. Senneville E, Legout L, Valette $M$, et al. Effectiveness and tolerability of prolonged linezolid treatment for chronic osteomyelitis: a retrospective study. Clin Ther 2006;28:1155-63.

10. Gerson SL, Kaplan SL, Bruss JB, et al. Hematologic effects of linezolid: summary of clinical experience. Antimicrob Agents Chemother 2002;46:2723-6.

11. Svensson EM, Dooley KE, Karlsson MO. Impact of lopinavir-ritonavir or nevirapine on bedaquiline exposures and potential implications for patients with tuberculosisHIV coinfection. Antimicrob Agents Chemother 2014;58:6406-12.

12. Xavier AS, Lakshmanan M. Delamanid: A new armor in combating drug-resistant tuberculosis. J Pharmacol Pharmacother 2014;5:222-4.

13. WHO Treatment Guidelines for DrugResistant Tuberculosis, 2016 Update. Available online: http://apps.who.int/iris/bitstream/hand le/10665/250125/9789241549639-eng.pdf?sequence=1

14. WHO. Companion Handbook to the WHO Guidelines for the Programmatic Management of Drug-Resistant Tuberculosis. Geneva, Switzerland: World Health Organization, 2014.

15. Falzon D, Jaramillo E, Schunemann HJ, et al. WHO guidelines for the programmatic management of drugresistant tuberculosis: 2011 update. Eur Respir J 2011;38:516-28.

16. Moodley R, Godec TR, Team ST. Short-course treatment for multidrug-resistant tuberculosis: the STREAM trials. Eur Respir Rev 2016;25:29-35.

17. Worley MV, Estrada SJ. Bedaquiline: a novel antitubercular agent for the treatment of multidrug-resistant tuberculosis. Pharmacotherapy 2014;34:1187-97.

18. Zhang C, Wang W, Zhou M, et al. The interaction of CD4 T-cell count and nevirapine hepatotoxicity in China: a change in national treatment guidelines may be warranted. J Acquir Immune Defic Syndr 2013;62:540-5.

19. De Lazzari E, Leon A, Arnaiz JA, et al. Hepatotoxicity of nevirapine in virologically suppressed patients according to gender and CD4 cell counts. HIV Med 2008;9:221-6.

20. Fortún J, Martin-Davila P, Navas E, et al. Linezolid for the treatment of multidrug-resistant tuberculosis. J Antimicrob Chemother 2005;56:180-5.

21. Anger HA, Dworkin F, Sharma S, et al. Linezolid use 
for treatment of multidrug-resistant and extensively drug-resistant tuberculosis, New York City, 2000-06. J Antimicrob Chemother 2010;65:775-83.

22. Koh WJ, Kang YR, Jeon K, et al. Daily $300 \mathrm{mg}$ dose of linezolid for multidrug-resistant and extensively drugresistant tuberculosis: updated analysis of 51 patients. J Antimicrob Chemother 2012;67:1503-7.

23. Sotgiu G, Centis R, D'Ambrosio L, et al. Efficacy, safety and tolerability of linezolid containing regimens in treating MDR-TB and XDR-TB: systematic review and metaanalysis. Eur Respir J 2012;40:1430-42.

24. Centers for Disease Control and Prevention. Provisional CDC guidelines for the use and safety monitoring of bedaquiline fumarate (Sirturo) for the treatment of multidrug-resistant tuberculosis. MMWR Recomm Rep 2013;62:1-12.

25. Mehrzad R, Barza M. Weighing the adverse cardiac effects of fluoroquinolones: A risk perspective. J Clin Pharmacol 2015;55:1198-206.

26. Stancampiano FF, Palmer WC, Getz TW, et al. Rare Incidence of Ventricular Tachycardia and Torsades de Pointes in Hospitalized Patients With Prolonged QT Who Later Received Levofloxacin: A Retrospective Study. Mayo Clin Proc 2015;90:606-12.

27. Im JH, Baek JH, Kwon HY, et al. Incidence and risk factors of linezolid-induced lactic acidosis. Int J Infect Dis 2015;31:47-52.

28. Roongruangpitayakul C, Chuchottaworn C. Outcomes of MDR/XDR-TB patients treated with linezolid: experience in Thailand. J Med Assoc Thai 2013;96:1273-82.

29. Isaakidis $P$, Casas EC, Das M, et al. Treatment outcomes for HIV and MDR-TB co-infected adults and children: systematic review and meta-analysis. Int J Tuberc Lung Dis 2015;19:969-78.

30. Liu AY, Yang Q, Huang Y, et al. Strong Relationship between Oral Dose and Tenofovir Hair Levels in a Randomized Trial: Hair as a Potential Adherence Measure for Pre-Exposure Prophylaxis (PrEP). PLoS One 2014;9:e83736.

31. Consolidated guidelines on the use of antiretroviral drugs for treating and preventing HIV infection: recommendations for a public health approach. 2 nd ed. Geneva: World Health Organization; 2016.

32. Sasabe H, Shimokawa Y, Shibata M, et al. Antitubercular Agent Delamanid and Metabolites as Substrates and Inhibitors of ABC and Solute Carrier Transporters. Antimicrob Agents Chemother 2016;60:3497-508.

33. UCSF. Database of Antiretroviral Drug Interactions. In:
Comprehensive, up-to-date information on HIV/AIDS treatment and prevention from the University of California San Francisco. 2017. Available online: http://hivinsite.ucsf. edu/insite? page $=$ ar-00-02 \& param $=235 \&$ post $=4$

34. Arentz M, Pavlinac P, Kimerling ME, et al. Use of antiretroviral therapy in tuberculosis patients on secondline anti-TB regimens: a systematic review. PLoS One 2012;7:e47370.

35. Padayatchi N, Abdool Karim SS, Naidoo K, et al. Improved survival in multidrug-resistant tuberculosis patients receiving integrated tuberculosis and antiretroviral treatment in the SAPiT Trial. Int J Tuberc Lung Dis 2014;18:147-54.

36. WHO. Guidelines for the programmatic management of drug-resistant tuberculosis. Geneva, Switzerland 2011. Report No.: WHO/HTM/TB/2009.420.

37. Abdool Karim SS, Naidoo K, Grobler A, et al. Integration of antiretroviral therapy with tuberculosis treatment. N Engl J Med 2011;365:1492-501.

38. Havlir DV, Kendall MA, Ive P, et al. Timing of antiretroviral therapy for HIV-1 infection and tuberculosis. N Engl J Med 2011;365:1482-91.

39. Blanc FX, Sok T, Laureillard D, et al. Earlier versus later start of antiretroviral therapy in $\mathrm{HIV}$-infected adults with tuberculosis. N Engl J Med 2011;365:1471-81.

40. Marais S, Pepper DJ, Marais BJ, et al. HIV-associated tuberculous meningitis--diagnostic and therapeutic challenges. Tuberculosis (Edinb) 2010;90:367-74.

41. Török ME, Yen NT, Chau TT, et al. Timing of initiation of antiretroviral therapy in human immunodeficiency virus (HIV)--associated tuberculous meningitis. Clin Infect Dis 2011;52:1374-83.

42. Andries K, Verhasselt P, Guillemont J, et al. A diarylquinoline drug active on the ATP synthase of Mycobacterium tuberculosis. Science 2005;307:223-7.

43. Sloan DJ, Davies GR, Khoo SH. Recent advances in tuberculosis: New drugs and treatment regimens. Curr Respir Med Rev 2013;9:200-10.

44. Diacon AH, Pym A, Grobusch MP, et al. Multidrugresistant tuberculosis and culture conversion with bedaquiline. N Engl J Med 2014;371:723-32.

45. Guglielmetti L, Le Du D, Jachym M, et al. Compassionate use of bedaquiline for the treatment of multidrug-resistant and extensively drug-resistant tuberculosis: interim analysis of a French cohort. Clin Infect Dis 2015;60:188-94.

46. Ndjeka N, Conradie F, Schnippel K, et al. Treatment of drug-resistant tuberculosis with bedaquiline in a high HIV prevalence setting: an interim cohort analysis. Int J Tuberc 
Lung Dis 2015;19:979-85.

47. van Heeswijk R, Diacon A, Pym A. The Pharmacokinetics (PK) Of Second Line Anti-tuberculosis Agents With And Without TMC207, An Investigational Antimycobacterial Agent, In Patients With MDR-TB [abstract]. 52nd Interscience Conference on Antimicrobial Agents and Chemotherapy (ICAAC). Sept 11, 2012. Abstract A-1258.

48. Svensson EM, Aweeka F, Park JG, et al. Model-based estimates of the effects of efavirenz on bedaquiline pharmacokinetics and suggested dose adjustments for patients coinfected with HIV and tuberculosis. Antimicrob Agents Chemother 2013;57:2780-7.

49. Pandie M, Wiesner L, McIlleron H, et al. Drug-drug interactions between bedaquiline and the antiretrovirals lopinavir/ritonavir and nevirapine in HIV-infected patients with drug-resistant TB. J Antimicrob Chemother 2016;71:1037-40.

50. Consolidated Guidelines on the Use of Antiretroviral Drugs for Treating and Preventing HIV Infection: Recommendations for a Public Health Approach. Geneva: World Health Organization; 2013.

51. Cox H, Ford N. Linezolid for the treatment of complicated drug-resistant tuberculosis: a systematic review and metaanalysis. Int J Tuberc Lung Dis 2012;16:447-54.

52. Lee M, Lee J, Carroll MW, et al. Linezolid for treatment of chronic extensively drug-resistant tuberculosis. N Engl J Med 2012;367:1508-18.

53. Hughes J, Isaakidis P, Andries A, et al. Linezolid for multidrug-resistant tuberculosis in HIV-infected and -uninfected patients. Eur Respir J 2015;46:271-4.

54. Vu DH, Bolhuis MS, Koster RA, et al. Dried blood spot analysis for therapeutic drug monitoring of linezolid in patients with multidrug-resistant tuberculosis. Antimicrob Agents Chemother 2012;56:5758-63.

55. Pea F, Furlanut M, Cojutti P, et al. Therapeutic drug monitoring of linezolid: a retrospective monocentric analysis. Antimicrob Agents Chemother 2010;54:4605-10.

56. Song T, Lee M, Jeon HS, et al. Linezolid Trough Concentrations Correlate with Mitochondrial ToxicityRelated Adverse Events in the Treatment of Chronic Extensively Drug-Resistant Tuberculosis. EBioMedicine 2015;2:1627-33

57. Tang S, Yao L, Hao X, et al. Efficacy, safety and tolerability of linezolid for the treatment of XDR-TB: a study in China. Eur Respir J 2015;45:161-70.

58. Moraza L, Leache L, Aquerreta I, et al. Linezolid-induced haematological toxicity. Farm Hosp 2015;39:320-6.

59. Sasaki T, Takane H, Ogawa K, et al. Population pharmacokinetic and pharmacodynamic analysis of linezolid and a hematologic side effect, thrombocytopenia, in Japanese patients. Antimicrob Agents Chemother 2011;55:1867-73.

60. De Vriese AS, Coster RV, Smet J, et al. Linezolid-induced inhibition of mitochondrial protein synthesis. Clin Infect Dis 2006;42:1111-7.

61. Bobylev I, Maru H, Joshi AR, et al. Toxicity to sensory neurons and Schwann cells in experimental linezolidinduced peripheral neuropathy. J Antimicrob Chemother 2016;71:685-91.

62. Bressler AM, Zimmer SM, Gilmore JL, et al. Peripheral neuropathy associated with prolonged use of linezolid. Lancet Infect Dis 2004;4:528-31.

63. Azamfirei L, Copotoiu SM, Branzaniuc K, et al. Complete blindness after optic neuropathy induced by short-term linezolid treatment in a patient suffering from muscle dystrophy. Pharmacoepidemiol Drug Saf 2007;16:402-4.

64. Libershteyn Y. Ethambutol/Linezolid Toxic Optic Neuropathy. Optom Vis Sci 2016;93:211-7.

65. Zhang X, Falagas ME, Vardakas KZ, et al. Systematic review and meta-analysis of the efficacy and safety of therapy with linezolid containing regimens in the treatment of multidrug-resistant and extensively drugresistant tuberculosis. J Thorac Dis 2015;7:603-15.

66. Zhanel GG, Love R, Adam H, et al. Tedizolid: a novel oxazolidinone with potent activity against multidrugresistant gram-positive pathogens. Drugs 2015;75:253-70.

67. Moran GJ, Fang E, Corey GR, et al. Tedizolid for 6 days versus linezolid for 10 days for acute bacterial skin and skin-structure infections (ESTABLISH-2): a randomised, double-blind, phase 3, non-inferiority trial. Lancet Infect Dis 2014;14:696-705.

68. Ong V, Flanagan S, Fang E, et al. Absorption, distribution, metabolism, and excretion of the novel antibacterial prodrug tedizolid phosphate. Drug Metab Dispos 2014;42:1275-84.

69. Schlosser MJ, Hosako H, Radovsky A, et al. Lack of neuropathological changes in rats administered tedizolid phosphate for nine months. Antimicrob Agents Chemother 2015;59:475-81.

70. Flanagan S, Passarell J, Lu Q, et al. Tedizolid population pharmacokinetics, exposure response, and target attainment. Antimicrob Agents Chemother 2014;58:6462-70.

71. Lemaire S, Tulkens PM, Van Bambeke F. Cellular pharmacokinetics of the novel biaryloxazolidinone radezolid in phagocytic cells: studies with macrophages and polymorphonuclear neutrophils. Antimicrob Agents 
Chemother 2010;54:2540-8.

72. Williams KN, Brickner SJ, Stover CK, et al. Addition of PNU-100480 to first-line drugs shortens the time needed to cure murine tuberculosis. Am J Respir Crit Care Med 2009;180:371-6.

73. Wallis RS, Dawson R, Friedrich SO, et al. Mycobactericidal activity of sutezolid (PNU-100480) in sputum (EBA) and blood (WBA) of patients with pulmonary tuberculosis. PLoS One 2014;9:e94462.

74. Louie A, Eichas K, Files K, et al. Activities of PNU100480 (PNU 480) alone, PNU 480 plus its major metabolite PNU-101603 (PNU 1603) and PNU 480 plus PNU 1603 in combination with rifampin (RIF) against Mycobacterium tuberculosis: Comparison with linezolid. ICAAC 51; 2011.

75. Matsumoto M, Hashizume H, Tomishige T, et al. OPC67683, a nitro-dihydro-imidazooxazole derivative with promising action against tuberculosis in vitro and in mice. PLoS Med 2006;3:e466.

76. Gler MT, Skripconoka V, Sanchez-Garavito E, et al. Delamanid for multidrug-resistant pulmonary tuberculosis. N Engl J Med 2012;366:2151-60.

77. Skripconoka V, Danilovits M, Pehme L, et al. Delamanid improves outcomes and reduces mortality in multidrugresistant tuberculosis. Eur Respir J 2013;41:1393-400.

78. Gupta R, Geiter LJ, Wells CD, et al. Delamanid for Extensively Drug-Resistant Tuberculosis. N Engl J Med 2015;373:291-2.

79. European Medicines Agency. Delamnid (Deltyba): Summary of Product Characteristics. London: Committee for Medicinal Products for Human Use (CHMP), 2014.

80. Shah S, Smith CJ, Lampe F, et al. Haemoglobin and albumin as markers of HIV disease progression in the highly active antiretroviral therapy era: relationships with gender. HIV Med 2007;8:38-45.

81. Feldman JG, Burns DN, Gange SJ, et al. Serum albumin as a predictor of survival in $\mathrm{HIV}$-infected women in the Women's Interagency HIV study. AIDS 2000;14:863-70.

82. Alvarez-Uria G, Midde M, Pakam R, et al. Diagnostic and Prognostic Value of Serum Albumin for Tuberculosis in HIV Infected Patients Eligible for Antiretroviral Therapy: Datafrom an HIV Cohort Study in India. Bioimpacts 2013;3:123-8.

83. Mallikaarjun S, Wells C, Petersen C, et al. Delamanid Coadministered with Antiretroviral Drugs or Antituberculosis Drugs Shows No Clinically Relevant Drug-Drug Interactions in Healthy Subjects. Antimicrob Agents Chemother 2016;60:5976-85.
84. Diacon AH, Dawson R, Hanekom M, et al. Early bactericidal activity of delamanid (OPC-67683) in smearpositive pulmonary tuberculosis patients. Int J Tuberc Lung Dis 2011;15:949-54.

85. Laliberté F, Bookhart BK, Nelson WW, et al. Impact of once-daily versus twice-daily dosing frequency on adherence to chronic medications among patients with venous thromboembolism. Patient 2013;6:213-24.

86. Hafkin J, Frias M, Hesseling A, et al. Pharmacokinetics and safety of peadiatric MDR-TB patients, aged 6-17 years. San Diego, CA, USA: ICAAC, 17-25 September 2015.

87. Sugarman J, Colvin C, Moran AC, et al. Tuberculosis in pregnancy: an estimate of the global burden of disease. Lancet Glob Health 2014;2:e710-6.

88. Mathad JS, Gupta A. Tuberculosis in pregnant and postpartum women: epidemiology, management, and research gaps. Clin Infect Dis 2012;55:1532-49.

89. Nachega J, Coetzee J, Adendorff T, et al. Tuberculosis active case-finding in a mother-to-child HIV transmission prevention programme in Soweto, South Africa. AIDS 2003;17:1398-400.

90. Singh N, Perfect JR. Immune reconstitution syndrome and exacerbation of infections after pregnancy. Clin Infect Dis 2007;45:1192-9.

91. Jana N, Vasishta K, Jindal SK, et al. Perinatal outcome in pregnancies complicated by pulmonary tuberculosis. Int J Gynaecol Obstet 1994;44:119-24.

92. Adhikari M. Tuberculosis and tuberculosis/HIV coinfection in pregnancy. Semin Fetal Neonatal Med 2009; 14:234-40.

93. Tabarsi P, Moradi A, Baghaei P, et al. Standardised secondline treatment of multidrug-resistant tuberculosis during pregnancy. Int J Tuberc Lung Dis 2011;15:547-50.

94. Palacios E, Dallman R, Munoz M, et al. Drug-resistant tuberculosis and pregnancy: treatment outcomes of 38 cases in Lima, Peru. Clin Infect Dis 2009;48:1413-9.

95. Caminero JA. Guidelines for Clinical and Operational Management of Drug-Resistant Tuberculosis. Paris, France: International Union Against Tuberculosis and Lung Disease, 2013.

96. Best-practice statement on the off-label use of bedaquiline and delamanid for the treatment of multidrug-resistant tuberculosis. World Health Organization2017 Contract No.: WHO/HTM/TB/2017.20.

97. Falzon D, Gandhi N, Migliori GB, et al. Resistance to fluoroquinolones and second-line injectable drugs: impact on multidrug-resistant TB outcomes. Eur Respir J 2013;42:156-68. 
98. Food and Drug Administration (U.S.). Highlights of prescribing information for Sirturo (bedaquiline). 2012 December. Available online: http://www.accessdata.fda. gov/drugsatfda_docs/label/2012/204384s000lbl.pdf

99. Matzke GR, Halstenson CE, Keane WF. Hemodialysis elimination rates and clearance of gentamicin and tobramycin. Antimicrob Agents Chemother 1984;25:128-30.

100. Chang CH, Chen YF, Wu VC, et al. Acute kidney injury due to anti-tuberculosis drugs: a five-year experience in an aging population. BMC Infect Dis 2014;14:23.

101.Peloquin CA, Berning SE, Nitta AT, et al. Aminoglycoside toxicity: daily versus thrice-weekly dosing for treatment of mycobacterial diseases. Clin Infect Dis 2004;38:1538-44.

102. Ellard GA. Absorption, metabolism and excretion of pyrazinamide in man. Tubercle 1969;50:144-58.

103. Malone RS, Fish DN, Spiegel DM, et al. The effect of hemodialysis on isoniazid, rifampin, pyrazinamide, and ethambutol. Am J Respir Crit Care Med 1999;159:1580-4.

104. British Thoracic Society Standards of Care Committee and Joint Tuberculosis Committee, Milburn H, Ashman $\mathrm{N}$, et al. Guidelines for the prevention and management of Mycobacterium tuberculosis infection and disease in adult patients with chronic kidney disease. Thorax 2010;65:557-70.

105. Strauss I, Erhardt F. Ethambutol absorption, excretion and dosage in patients with renal tuberculosis. Chemotherapy 1970;15:148-57.

106. Malone RS, Fish DN, Spiegel DM, et al. The effect of hemodialysis on cycloserine, ethionamide, paraaminosalicylate, and clofazimine. Chest 1999;116:984-90.

107. Bowersox DW, Wintebauer RH, Stewart GL, et al. Isoniazid dosage in patients with renal failure. $\mathrm{N}$ Engl J Med 1973;289:84-7.

108. Fish DN, Chow AT. The clinical pharmacokinetics of levofloxacin. Clin Pharmacokinet 1997;32:101-19.

109. Fillastre JP, Montay G, Bruno R, et al. Pharmacokinetics of sparfloxacin in patients with renal impairment. Antimicrob Agents Chemother 1994;38:733-7.

110. Coyne KM, Pozniak AL, Lamorde M, et al. Pharmacology of second-line antituberculosis drugs and potential for interactions with antiretroviral agents. AIDS 2009;23:437-46.

111. Galløe AM, Graudal N, Christensen HR, et al. Aminoglycosides: single or multiple daily dosing? A metaanalysis on efficacy and safety. Eur J Clin Pharmacol 1995;48:39-43.

112.Labarga P, Barreiro P, Martin-Carbonero L, et al. Kidney tubular abnormalities in the absence of impaired glomerular function in HIV patients treated with tenofovir. AIDS 2009;23:689-96.
113. Reid A, Stohr W, Walker AS, et al. Severe renal dysfunction and risk factors associated with renal impairment in $\mathrm{HIV}$-infected adults in Africa initiating antiretroviral therapy. Clin Infect Dis 2008;46:1271-81.

114.Kasembeli AN, Duarte R, Ramsay M, et al. African origins and chronic kidney disease susceptibility in the human immunodeficiency virus era. World J Nephrol 2015;4:295-306.

115. Bigé N, Lanternier F, Viard JP, et al. Presentation of HIVassociated nephropathy and outcome in HAART-treated patients. Nephrol Dial Transplant 2012;27:1114-21.

116. Williams DI, Williams DJ, Williams IG, et al. Presentation, pathology, and outcome of HIV associated renal disease in a specialist centre for HIV/AIDS. Sex Transm Infect 1998;74:179-84.

117. Ramsuran D, Bhimma R, Ramdial PK, et al. The spectrum of HIV-related nephropathy in children. Pediatr Nephrol 2012;27:821-7.

118. Katiyar SK, Bihari S, Prakash S, et al. A randomised controlled trial of high-dose isoniazid adjuvant therapy for multidrug-resistant tuberculosis. Int J Tuberc Lung Dis 2008;12:139-45.

119. Wong WM, Wu PC, Yuen MF, et al. Antituberculosis drug-related liver dysfunction in chronic hepatitis B infection. Hepatology 2000;31:201-6.

120. Patel PA, Voigt MD. Prevalence and interaction of hepatitis B and latent tuberculosis in Vietnamese immigrants to the United States. Am J Gastroenterol 2002;97:1198-203.

121.Panel on Antiretroviral Guidelines for Adults and Adolescents. Guidelines for the use of antiretroviral agents in HIV-1-infected adults and adolescents. Department of Health and Human Services. Available online: http:// aidsinfo.nih.gov/ContentFiles/AdultandAdolescentGL. pdf, Accessed on 5th of October 2017.

122. Panel on Opportunistic Infections in HIV-Infected Adults and Adolescents. Guidelines for the prevention and treatment of opportunistic infections in HIV-infected adults and adolescents: recommendations from the Centers for Disease Control and Prevention, the National Institutes of Health, and the HIV Medicine Association of the Infectious Diseases Society of America. Available online: http://aidsinfo.nih.gov/contentfiles/lvguidelines/ adult_oi.pdf. Accessed 5th October 2017.

123. Rathmann W, Giani G. Global prevalence of diabetes: estimates for the year 2000 and projections for 2030. Diabetes Care 2004;27:2568-9; author reply 9. 124.Jeon CY, Murray MB. Diabetes mellitus increases the 
risk of active tuberculosis: a systematic review of 13 observational studies. PLoS Med 2008;5:e152.

125. Stevenson CR, Critchley JA, Forouhi NG, et al. Diabetes and the risk of tuberculosis: a neglected threat to public health? Chronic Illn 2007;3:228-45.

126. Baker MA, Harries AD, Jeon CY, et al. The impact of diabetes on tuberculosis treatment outcomes: a systematic review. BMC Med 2011;9:81.

127.Hung CL, Chien JY, Ou CY. Associated factors for tuberculosis recurrence in Taiwan: a nationwide nested case-control study from 1998 to 2010. PLoS One 2015;10:e0124822.

128.Jiménez-Corona ME, Cruz-Hervert LP, Garcia-Garcia L, et al. Association of diabetes and tuberculosis: impact on treatment and post-treatment outcomes. Thorax 2013;68:214-20.

129. Dooley KE, Chaisson RE. Tuberculosis and diabetes mellitus: convergence of two epidemics. Lancet Infect Dis 2009;9:737-46.

130. Fisher-Hoch SP, Whitney E, McCormick JB, et al. Type 2 diabetes and multidrug-resistant tuberculosis. Scand J Infect Dis 2008;40:888-93.

131.Pérez-Guzmán C, Vargas MH, Torres-Cruz A, et al. Diabetes modifies the male:female ratio in pulmonary tuberculosis. Int J Tuberc Lung Dis 2003;7:354-8.

132. Restrepo BI, Schlesinger LS. Impact of diabetes on the natural history of tuberculosis. Diabetes Res Clin Pract 2014;106:191-9.

133. Salindri AD, Kipiani M, Kempker RR, et al. Diabetes Reduces the Rate of Sputum Culture Conversion in Patients With Newly Diagnosed Multidrug-Resistant Tuberculosis. Open Forum Infect Dis 2016;3:ofw126.

134.Pérez-Navarro LM, Fuentes-Dominguez FJ, ZentenoCuevas R. Type 2 diabetes mellitus and its influence in the development of multidrug resistance tuberculosis in patients from southeastern Mexico. J Diabetes Complications 2015;29:77-82.

135. Baghaei P, Tabarsi P, Javanmard P, et al. Impact of diabetes mellitus on tuberculosis drug resistance in new cases of tuberculosis. J Glob Antimicrob Resist 2016;4:1-4.

136. American Diabetes Association. Standards of medical care in diabetes--2014. Diabetes Care 2014;37 Suppl 1:S14-80.

137.Kim PS, Woods C, Georgoff P, et al. A1C underestimates glycemia in HIV infection. Diabetes Care 2009;32:1591-3.

138. Diop ME, Bastard JP, Meunier N, et al. Inappropriately low glycated hemoglobin values and hemolysis in HIV-infected patients. AIDS Res Hum Retroviruses 2006;22:1242-7.
139. Slama L, Palella FJ Jr, Abraham AG, et al. Inaccuracy of haemoglobin A1c among HIV-infected men: effects of CD4 cell count, antiretroviral therapies and haematological parameters. J Antimicrob Chemother 2014;69:3360-7.

140. Niemi M, Backman JT, Fromm MF, et al. Pharmacokinetic interactions with rifampicin: clinical relevance. Clin Pharmacokinet 2003;42:819-50.

141. Tornio A, Niemi M, Neuvonen PJ, et al. Drug interactions with oral antidiabetic agents: pharmacokinetic mechanisms and clinical implications. Trends Pharmacol Sci 2012;33:312-22.

142. de Steenwinkel JE, Aarnoutse RE, de Knegt GJ, et al. Optimization of the rifampin dosage to improve the therapeutic efficacy in tuberculosis treatment using a murine model. Am J Respir Crit Care Med 2013;187:1127-34.

143. Scheen AJ, Paquot N. Metformin revisited: a critical review of the benefit-risk balance in at-risk patients with type 2 diabetes. Diabetes Metab 2013;39:179-90.

144. Heller S, Darpo B, Mitchell MI, et al. Considerations for assessing the potential effects of antidiabetes drugs on cardiac ventricular repolarization: A report from the Cardiac Safety Research Consortium. Am Heart J 2015;170:23-35.

145. Tolman KG, Chandramouli J. Hepatotoxicity of the thiazolidinediones. Clin Liver Dis 2003;7:369-79, vi.

146. Ruslami R, Aarnoutse RE, Alisjahbana B, et al. Implications of the global increase of diabetes for tuberculosis control and patient care. Trop Med Int Health 2010;15:1289-99.

147. Chiang CY, Lee JJ, Chien ST, et al. Glycemic control and radiographic manifestations of tuberculosis in diabetic patients. PLoS One 2014;9:e93397.

148. Niazi AK, Kalra S. Diabetes and tuberculosis: a review of the role of optimal glycemic control. J Diabetes Metab Disord 2012;11:28.

149. Rodbard HW, Jellinger PS, Davidson JA, et al. Statement by an American Association of Clinical Endocrinologists/ American College of Endocrinology consensus panel on type 2 diabetes mellitus: an algorithm for glycemic control. Endocr Pract 2009;15:540-59.

150. Riza AL, Pearson F, Ugarte-Gil C, et al. Clinical management of concurrent diabetes and tuberculosis and the implications for patient services. Lancet Diabetes Endocrinol 2014;2:740-53.

151. Wang JY, Lee MC, Shu CC, et al. Optimal duration of anti-TB treatment in patients with diabetes: nine or six months? Chest 2015;147:520-8.

152. Lee PH, Lin HC, Huang AS, et al. Diabetes and risk of tuberculosis relapse: nationwide nested case-control study. 
PLoS One 2014;9:e92623.

153. Calligaro GL, Theron G, Khalfey H, et al. Burden of tuberculosis in intensive care units in Cape Town, South Africa, and assessment of the accuracy and effect on patient outcomes of the Xpert MTB/RIF test on tracheal aspirate samples for diagnosis of pulmonary tuberculosis: a prospective burden of disease study with a nested randomised controlled trial. Lancet Respir Med 2015;3:621-30.

154. Heysell SK, Moore JL, Peloquin CA, et al. Outcomes and use of therapeutic drug monitoring in multidrug-resistant tuberculosis patients treated in virginia, 2009-2014. Tuberc Respir Dis (Seoul) 2015;78:78-84.

155.Alsultan A, Peloquin CA. Therapeutic drug monitoring in the treatment of tuberculosis: an update. Drugs 2014;74:839-54.

156. Centers for Disease Control and Prevention: HIV surveillance reports. HIV Surveillance Report, 2016; vol. 28. Available online: http://www.cdc.gov/hiv/library/ reports/hiv-surveillance.html. Published November 2017.

Cite this article as: Esmail A, Sabur NF, Okpechi I, Dheda K. Management of drug-resistant tuberculosis in special subpopulations including those with HIV co-infection, pregnancy, diabetes, organ-specific dysfunction, and in the critically ill. J Thorac Dis 2018;10(5):3102-3118. doi: 10.21037/ jtd.2018.05.11 